\title{
VEGF-C gene therapy augments postnatal lymphangiogenesis and ameliorates secondary lymphedema
}

\author{
Young-sup Yoon, ${ }^{1}$ Toshinori Murayama, ${ }^{1}$ Edwin Gravereaux, ${ }^{1}$ Tengiz Tkebuchava, ${ }^{1}$ \\ Marcy Silver, ${ }^{1}$ Cynthia Curry, ${ }^{1}$ Andrea Wecker, ${ }^{1}$ Rudolf Kirchmair, ${ }^{1}$ Chun Song Hu, ${ }^{1}$ \\ Marianne Kearney, ${ }^{1}$ Alan Ashare, ${ }^{2}$ David G. Jackson, ${ }^{3}$ Hajime Kubo, ${ }^{4}$ Jeffrey M. Isner, ${ }^{1}$ \\ and Douglas W. Losordo ${ }^{1}$
}

${ }^{1}$ Department of Vascular Medicine and Department of Cardiovascular Research, and ${ }^{2}$ Department of Nuclear Medicine, St. Elizabeth's Medical Center, Tufts University School of Medicine, Boston, Massachusetts, USA

${ }^{3}$ Molecular Immunology Group, Institute of Molecular Medicine, University of Oxford, Oxford, United Kingdom ${ }^{4}$ Molecular/Cancer Biology Laboratory, Haartman Institute, University of Helsinki, Helsinki, Finland

\begin{abstract}
Although lymphedema is a common clinical condition, treatment for this disabling condition remains limited and largely ineffective. Recently, it has been reported that overexpression of VEGF-C correlates with increased lymphatic vessel growth (lymphangiogenesis). However, the effect of VEGF-C-induced lymphangiogenesis on lymphedema has yet to be demonstrated. Here we investigated the impact of local transfer of naked plasmid DNA encoding human VEGF-C (phVEGF-C) on two animal models of lymphedema: one in the rabbit ear and the other in the mouse tail. In a rabbit model, following local phVEGF-C gene transfer, VEGFR-3 expression was significantly increased. This gene transfer led to a decrease in thickness and volume of lymphedema, improvement of lymphatic function demonstrated by serial lymphoscintigraphy, and finally, attenuation of the fibrofatty changes of the skin, the final consequences of lymphedema. The favorable effect of phVEGF-C on lymphedema was reconfirmed in a mouse tail model. Immunohistochemical analysis using lymphatic-specific markers: VEGFR-3, lymphatic endothelial hyaluronan receptor-1, together with the proliferation marker $\mathrm{Ki}-67 \mathrm{Ab}$ revealed that $\mathrm{phVEGF-C}$ transfection potently induced new lymphatic vessel growth. This study, we believe for the first time, documents that gene transfer of phVEGF-C resolves lymphedema through direct augmentation of lymphangiogenesis. This novel therapeutic strategy may merit clinical investigation in patients with lymphedema.
\end{abstract}

J. Clin. Invest. 111:717-725 (2003). doi:10.1172/JCI200315830.

\section{Introduction}

Lymphedema is defined as the progressive accumulation of protein-rich fluid in the interstitial spaces that results from an anatomic or functional obstruction in the lymphatic system (1). While primary lymphedema occurs infrequently on a hereditary or idiopathic basis, secondary lymphedema is common worldwide, primarily due to the increase in radical surgery and radiotherapy for cancer in developed countries and infectious disease (filariasis) in developing countries $(2,3)$. Despite substantial advances in both surgical and con-

Received for publication April 30, 2002, and accepted in revised form January 7, 2003.

Address correspondence to: Douglas W. Losordo, Cardiovascular Research, St. Elizabeth's Medical Center, 736 Cambridge Street, Boston, Massachusetts 02135, USA. Phone: (617) 789-3346; Fax: (617) 779-6362;

E-mail: douglas.losordo@tufts.edu.

Conflict of interest: The authors have declared that no conflict of interest exists.

Nonstandard abbreviations used: naked plasmid DNA encoding human VEGF-C (phVEGF-C); coefficient of variation (cv); lymphatic endothelial hyaluronan receptor-1 (LYVE-1); platelet endothelial cell adhesion molecule-1 (PECAM-1). servative techniques, therapeutic options for management of lymphedema are limited $(3,4)$. Pathophysiologically, restoration of the lymph-transporting capacity would appear to represent the optimal treatment for lymphedema. However, no means for accomplishing new lymphatic channel development currently exists. Growth of new lymphatic vessels (lymphangiogenesis) in healthy animals is rapid. The best example of the natural recovery of lymphatic drainage in animals is the complete restoration of lymphatic flow after limb reimplantation (5-9). Therefore the primary difficulty found in lymphedema animal models is to develop a method to sustain lymphedema long enough to allow evaluation of therapies.

Recent molecular studies have begun to elucidate the basis for lymphangiogenesis that can be stimulated by various cytokines, including VEGF-C (VEGF-2) (10, 11). VEGF-C, the first ligand to be discovered for VEGFR-3 (Flt4), is a member of the VEGF family of polypeptide growth factors. VEGF-C binds to endothelial cell receptors VEGFR-2 (Flk1) and VEGFR-3 (12-15). Although VEGFR-3 plays a critical role for both vascular and lymphatic endothelial cell develop- 

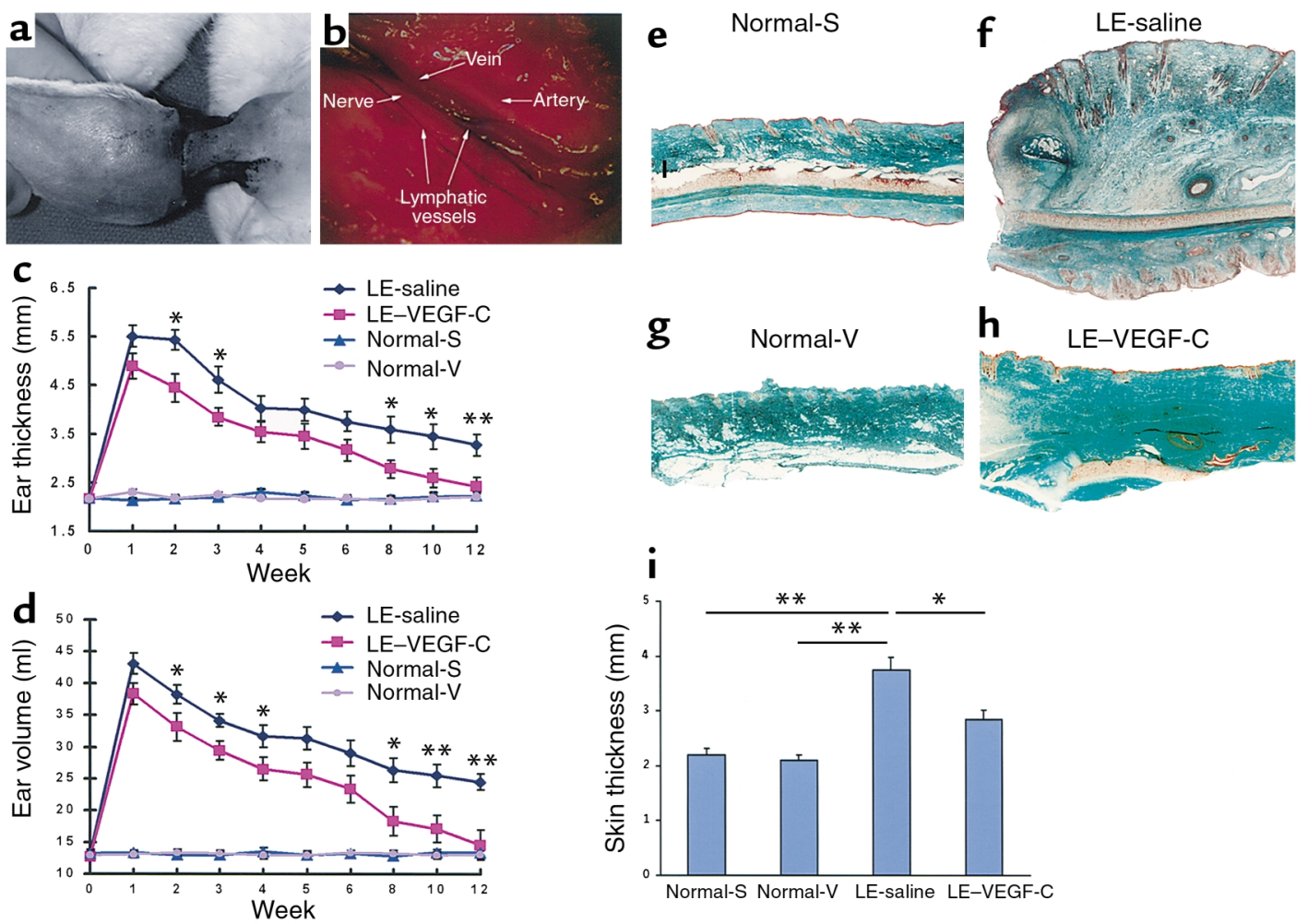

\section{Figure 1}

(a-d) Rabbit ear model of lymphedema: effect of phVEGF-C gene therapy. (a) Postoperative appearance of the dorsal surface of the rabbit ear. Lymphedema surgery leaves a gap of cartilage crossed only by the skin bridge. (b) A view under a surgical microscope after lifting up the skin bridge showing neurovascular bundle. Lymphatic vessels were visualized as blue lines (arrows) due to the uptake of Evans blue. Ear thickness $(\mathbf{c})$ and volume $(\mathbf{d})$ show consistent differences between the VEGF-C and saline groups over 12 weeks. ${ }^{*} P<0.05 ;{ }^{*} P<0.01$. (e-i) Decreased skin thickness after phVEGF-C transfer in a rabbit lymphedema model. Photos show cross sections of the skin after elastic-tissue trichrome staining 8 weeks after lymphedema surgery. Compared with normal ears (e and $\mathbf{g})$, operated ears (f $\mathbf{f}$ and $\mathbf{h})$ had fibrofatty tissue deposition and thus greater skin thickness. The phVEGF-C-transfected ear shown in $\mathbf{h}$ shows less fibrosis and decreased thickness compared with the saline-injected ear (f), which demonstrates other characteristic features of lymphedema, such as profound epidermal hyperplasia and papillomatosis. (i) Measurement of skin thickness from histologic sections shows a significant difference between the saline and VEGF-C groups $(P<0.05)$. ${ }^{*} P<0.05 ;{ }^{*} P<0.01$. Scale bar, $500 \mu \mathrm{m}$. Normal-S and Normal-V indicate unoperated ears from the saline and VEGF-C groups, respectively; LE indicates lymphedema-operated ears.

ment, its expression becomes limited to the lymphatic endothelium beginning in the late stages of development (16-18). Overexpression of VEGF-C cDNA in the skin of transgenic mice induced lymphatic endothelial cell proliferation and hyperplasia of the lymphatic vasculature, and recombinant VEGF-C specifically stimulated lymphangiogenesis in chorioallantoic membrane $(11,19)$. Recently, direct evidence of the link between VEGFR-3 and lymphedema has been found: it has been reported that human hereditary lymphedema is associated with heterozygous missense mutation of the Flt4 gene, which leads to insufficient VEGFR-3 signaling $(20,21)$. Recently, it was demonstrated that subcutaneous injection of adenovirus or adeno-associated virus encoding VEGF-C could generate lymphatic vessels in the skin of normal mice (22) and in a mouse model (chy mouse) of primary lymphedema (23). Although these studies showed that VEGF-C could induce lymphangiogenesis in vivo, they failed to show that this VEGFC-induced lymphangiogenesis could improve overall lymphatic vascular dysfunction and prevent chronic changes accompanied by lymphedema, which are the key determinants of whether VEGF-C can be used as a therapeutic option to treat human lymphedema.

Accordingly, first we sought to establish reliable animal models of secondary lymphedema. The two animal models used here provided complementary measurements: the rabbit ear had the advantage of size, which is conducive to direct measurements and lymphoscintigraphy, whereas the mouse tail had advantages for immunohistochemistry owing to the availability of lymphatic vessel-specific Ab's. Next, using these animal models, we investigated whether local transfer of naked plasmid DNA encoding human VEGF-C (phVEGF-C) could promote lymphangiogenesis and improve physical, functional, and pathologic aspects of lymphedema.

\section{Methods}

All animal protocols were approved by the Institutional Animal Care and Use Committee of St. Elizabeth's Medical Center. Investigators for the follow-up examinations were blinded to the identity of the treatment given. 
Rabbit ear model of lymphedema. We modified several previous rabbit ear models to overcome the shortcomings of rapid lymphatic regeneration and to provide the bed for new lymphatic vessel growth $(5,24,25)$. To meet those requirements, we used old (3-4 years of age) New Zealand White rabbits and created a skin bridge. Before the operation, the lymphatic vessels were identified by intradermal injection of $0.2 \mathrm{ml}$ of $1 \%$ Evans blue dye at the dorsal tip of the right ear. A strip of skin, subcutaneous tissues, and perichondrium $3 \mathrm{~cm}$ wide was circumferentially excised from the base of the ear, except for the central portion ( $1 \mathrm{~cm}$ in width) of the dorsal skin, i.e., a "skin bridge" underneath which runs the neurovascular bundle (Figure 1b). After the distal edge of the skin bridge was incised, lymphatic channels were dissected and the lymphatic stumps were resected under a dissecting microscope. Other edges of skin were inversely sutured to the perichondrium to prevent reapproximation of skin edges and recanalization of the lymphatic vessels. This created a strip of bare cartilage, leaving only the skin bridge for lymphatic growth (Figure 1a).

Preparation of phVEGF-C and gene transfer protocol in a rabbit model. A total of 54 rabbits was randomized into two groups in a blinded fashion for treatment with phVEGF-C or control (saline). In the VEGF-C group, $500 \mu \mathrm{g}$ of phVEGF-C in $0.5 \mathrm{ml}$ volume was injected intradermally and subcutaneously at the skin bridge using a 27 gauge needle on days 1,6 , and 11 after lymphedema surgery.

Measurement of ear thickness and volume. The thickness of the rabbit ears was measured $1 \mathrm{~cm}$ medial and distal to the medial border of the skin bridge with a vernier caliper. The ear was put in a 50-ml cylinder filled with water. After removing the ear, the volume of water displaced by the ear was measured (25). The thickness and volume of all ears was measured before surgery and every week for 6 weeks, and thereafter every 2 weeks until the 12 -week point ( $n=12$ in each group). Measurement of skin thickness in bistologic sections. Thickness of the ear skin at 8 weeks after surgery was measured in a cross section of the skin just distal to the skin bridge in paraffin-embedded histologic specimens after elastictissue trichrome staining as described previously (26) $n=5$ in each group). Lymphoscintigraphy and quantitative analysis. Tc-99m-filtered sulfur colloid was injected intradermally into the dorsal tip of rabbit ears at a dose

\section{Figure 2}

of $50 \mu \mathrm{Ci}$. Imaging was performed using a large-fieldof-view Genesys $\gamma$ camera (ADAC Laboratories, Milpitas, California, USA).

To quantitatively compare lymphatic drainage of the injected radiotracers, radioactivity within the ears was counted. The $\gamma$ counts at injection sites were similar in both ears of the saline and VEGF-C groups $(P=0.93)$. For standardization, the ratio of radioactivity of the operated ear to that of the normal (contralateral) ear, designated the radioactivity index, was used to compare lymphatic drainage at 4, 8, and 12 weeks (see Figure 2, g-i). The validity of the radioactivity index was verified by repeated examination of normal ears $(n=7)$ for intraindividual variation, which was $6 \%$ (coefficient of variation $[\mathrm{cv} \%]$ ), and by comparison of the day 1 postoperative lymphoscintigrams for interindividual variation $(n=20)$, which was $10 \%$ (cv \%).

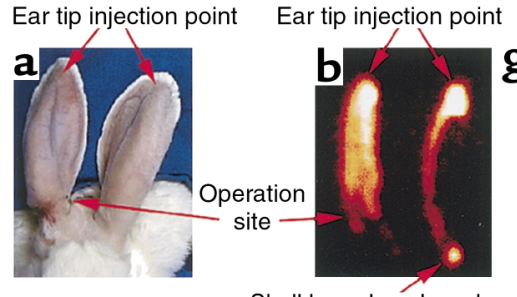

Skull base lymph node
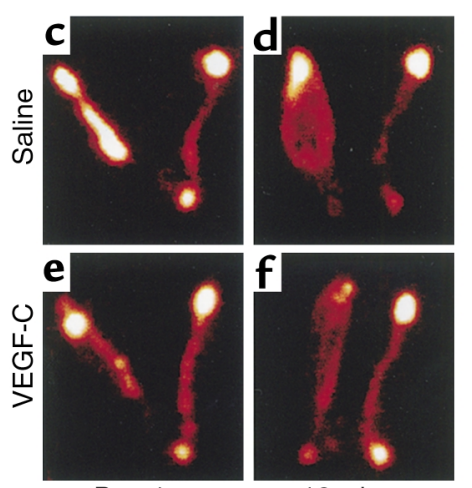

12 wks

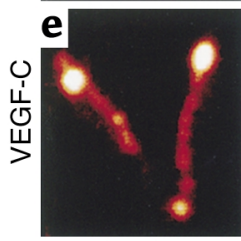

Day 1

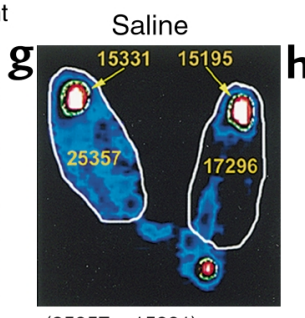
$\frac{(25357-15331)}{(17296-15195)}=4.77$
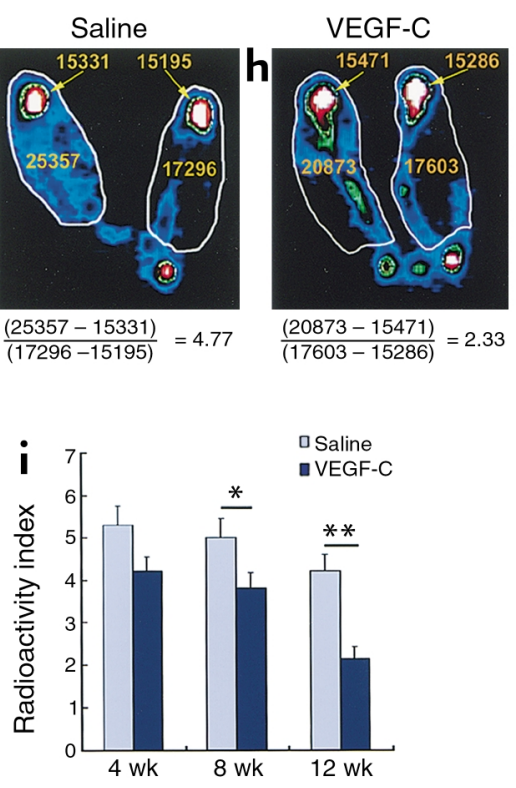

Temporal changes of lymphatic function visualized by lymphoscintigraphy. (a and b) Orientation of the lymphoscintigraphic images. In normal ears, lymphatic flow assumes a linear pattern and the draining LNs are clearly visible. In the operated ear, the lymphatic passages were blocked, resulting in backward diffusion and no visualization of LNs. (c and d) Temporal changes in the saline group. Even at 12 weeks (d), lymphoscintigraphy demonstrates substantial impairment of lymphatic drainage of the saline-injected ear, indicated by dermal backflow and faint visualization of the LNs. (e and $\mathbf{f}$ ) Temporal changes in the VEGF-C group. In the phVEGF-C-transfected ears, there was remarkable improvement of draining function. At 12 weeks, a linear passage of radiotracer, decreased dermal backflow, and increased uptake by LNs were observed. ( $g$ and $\mathbf{h}$ ) Representative lymphoscintigraphic images and calculation of radioactivity index from the saline $(\mathbf{g})$ and VEGF-C group (h). To quantitatively compare lymphatic drainage, the radioactivity within the ear was counted. Net radioactivity of the ear was obtained by subtracting $\gamma$ counts at injection sites (arrows) from the total counts of the ear. The radioactivity index is the ratio of radioactivity of the operated ear divided by the radioactivity of the normal ear; this was used to compare lymphatic drainage function of the lymphedema ears. Higher ratios indicate more persistent radioactivity and less lymphatic drainage. (i) Comparison between the saline and VEGF-C groups shows the values were consistently lower in the VEGF-C group at 4,8 , and 12 weeks. ${ }^{*} P<0.05 ;{ }^{*} P<0.01$. 


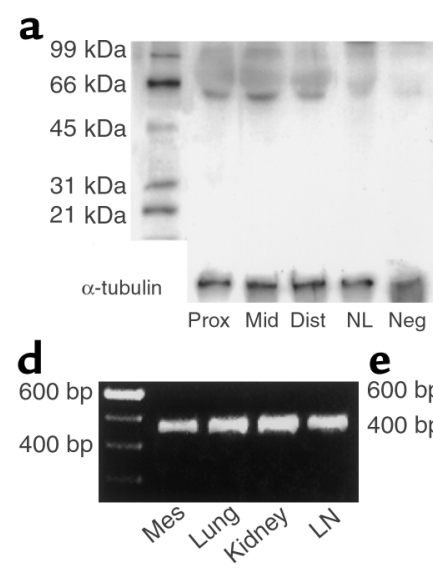

h

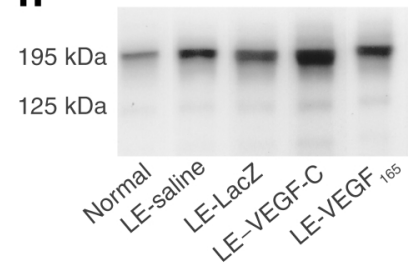

b

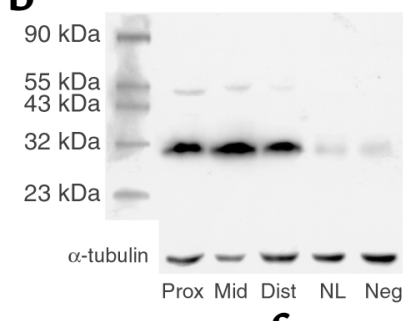

C

$\begin{array}{lr}\mathrm{Rb} & \\ \mathrm{Bo} & 1 \\ \mathrm{Hu} & 1 \\ \mathrm{Mo} & \mathrm{I} \\ \mathrm{Rb} & 51 \\ \mathrm{Bo} & 51 \\ \mathrm{Hu} & 51 \\ \mathrm{Mo} & 51 \\ \mathrm{Rb} & 101 \\ \mathrm{Bo} & 101 \\ \mathrm{Hu} & 101 \\ \mathrm{Mo} & 101\end{array}$

ACAYAGRHVP SI WWYKDEFL LQEESGIDLA DSNQRLSIQR YREEDAGRYL RCPYAGTHNP SI WWYKDEKL LEEESGIDLA DSNQRLSIQR YREEDAGHYL DCL YAGKHAP SI YWYKDERL LEEKSGYDLA DSNOKLSIOR YREEDAGRYL RCPUAGAHUP SI YWYKDERL LEKESGIDLA DSNQRLSIQR YREEDAGRYL CSVCNAKGCY NSSASYAVgG AEDRGSMEIV ILVGTGVIAY FFWVLLLLIF CSVCNAKGCV NSSASVAVEG SEDKGSWEIV ILVGTGVIAY FFWVLLLLIF CSVCNAKGCV NSSASVAVEG SEDKGSMEIV ILVGTGVIAV FFWVLLLLIF CSUCNAKGCY NSSASVAVEG SEDKGSMEIV ILIGTGVIRY FFWVLLLLIF

CNMRRPAHAD IKTGYLSIIM DPGEVPLEEQ CEYLSYOASQ CNMRRPTHAD IKTGYLSI IM DPGEVPLEEQ CEYLSVDASQ CNMARPAHAD IKTGYLSI IM DPGEYPLEEQ CEYLSYDASQ CNMKRPAHAD IKTGYLSI IM DPGEVPLEEQ CEYLSYDASQ
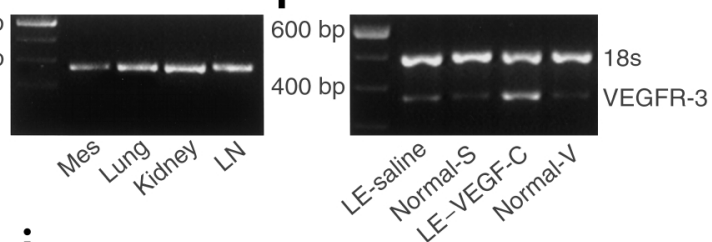

i

$235 \mathrm{kDa}$ $195 \mathrm{kDa}$

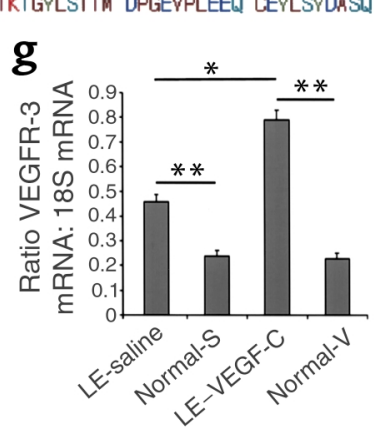

\section{Figure 3}

Increased expression of VEGF-C protein and VEGFR-3 mRNA in the phVEGF-C-transfected ears. (a and b) Western blot of VEGF-C protein from skin. VEGF-C was detected in its 58-kDa (a) and 31-kDa forms (b). VEGF-C protein expression was significantly higher at and around the phVEGF-C-transfected lymphedema skin. Prox, Mid, and Dist represent samples obtained from ear skin proximal to the skin bridge, skin from the bridge itself, and intact ear skin just distal to the skin bridge of the phVEGF-C-transfected ear, respectively. Neg, samples from the skin bridge of saline-injected lymphedema ear. NL, samples from the bridge site of unoperated contralateral ear. (c and $\mathbf{d}$ ) Using degenerate oligonucleotides, RT-PCR was performed for total RNA extracted from mesentery (Mes), lung, kidney, and LNs. The PCR product (470 bp) from the kidney sample was sequenced. At the protein level, the rabbit (Rb) VEGFR-3 clone displayed $92.9 \%, 93.6 \%$, and $94.3 \%$ identity with human ( $\mathrm{Hu}$ ), bovine (Bo), and mouse (Mo) VEGFR-3, respectively. (e) New primer sets were designed from the sequenced rabbit VEGFR-3 DNA, yielding a single PCR product of 362 bp. (f) Representative semiquantitative RT-PCR showing higher expression of VEGFR-3 in the lymphedema skin transfected with phVEGF-C than in the saline-injected or unoperated skin. (g) Quantification of VEGFR-3 mRNA levels. $\left({ }^{*} P<0.001\right.$. $\left.{ }^{*} P<0.01\right)$. (h and $\left.\mathbf{i}\right)$ The effect of phVEGF-C gene transfer on tyrosyl phosphorylation of VEGFR-3 (h) and VEGFR-2 (i) by immunoprecipitation with anti-phosphotyrosine Ab followed by Western blot analysis with anti-VEGFR-3 or anti-VEGFR-2 Ab's, respectively. Samples transfected with phVEGF-C revealed similar levels of phosphorylated VEGFR-2 compared with the control groups (saline and LacZ).

Western analysis of VEGF-C transgene expression in tissue. Samples harvested from the skin bridge and from tissue proximal and distal to the skin bridge of the operated ears, and from the bridge site of the contralateral ears were snap frozen in liquid nitrogen 7 days after the second injection of phVEGF-C (postoperative day 13) ( $n=5$ in each group). Western analysis was performed as described (27).

Molecular cloning of partial rabbit VEGFR-3 cDNA. Because the rabbit VEGFR-3 DNA sequence has not been identified, we sequenced part of the VEGFR-3 cDNA using degenerate oligonucleotides. Degenerate oligonucleotides were designed from conserved amino acid sequences NVSDSLEM and WEFPRER, located at the transmembrane domain of human and mouse VEGFR-3 (28, 29). The deduced oligonucleotide sequences were 5'-AACGTGAG (CT)GACTC (GC) (CT)T (AGCT)GA (AG)ATG-3' and 5'-CC (GT)YTC (CT)C (GT)GGG (AG)AA (CT)TCCCA-3', respectively. A single PCR product of $470 \mathrm{bp}$ was obtained from all the tissues (see Figure 3d).
Semiquantitative RT-PCR analysis of VEGFR-3. Using samples harvested from the bridge site of both ears at postoperative day 13 ( $n=5$ in each group), total RNA was isolated and RT-PCR was performed as described above. The primer pair used, designed on the basis of the sequenced cDNA's for rabbit VEGFR-3, was 5'-TATGGTACAAAGATGAGAGGC-3' (sense) and 5'-ACAGGTATTCACATTGCTCCT-3' (antisense). To quantify the VEGFR-3 mRNA, we used the "competimer" quantitative PCR technique (Ambion Inc., Austin, Texas, USA) according to the manufacturer's instructions. To the VEGFR-3 PCR mix, we added a mix of $18 \mathrm{~S}$ primer pairs and 18S 3'end modified primers (competimers) at a ratio of 1:9, yielding a 488-bp product. PCR was performed as follows: $94^{\circ} \mathrm{C}, 2 \mathrm{~min}$ (once); $94^{\circ} \mathrm{C}, 15 \mathrm{~s} ; 50^{\circ} \mathrm{C}, 30 \mathrm{~s} ; 72^{\circ} \mathrm{C}, 1$ $\min \left(40\right.$ cycles); $72^{\circ} \mathrm{C}, 10 \mathrm{~min}$ (once). PCR products were separated on $1.5 \%$ agarose gel and quantified by integrated density analysis software (EagleSight Software 3.2; Stratagene, La Jolla, California, USA).

Mouse tail model of lymphedema. Male nude (nu/nu) mice (Harlan, Indianapolis, Indiana, USA) 12 weeks of 
age were used. A mouse lymphedema model was created by modifying a previous model (30).

Gene transfer protocol in a mouse tail model. In total, 115 mice were randomized into five groups: no operation, VEGF-C, VEGF 165 , LacZ, and saline $(n=23$ in each group). The unoperated group served as negative control. The other groups underwent the operation as described. In the VEGF-C group, $100 \mu \mathrm{g}$ of phVEGF-C was given in $100 \mu \mathrm{l}$ volume on days 1,6 , and 11 after the operation, respectively. The phVEGF $_{165}$ plasmid $(18,31)$, pGSV-nlsLacZ (32) (a nuclear targeted LacZ gene plasmid encoding the protein $\beta$-galactosidase), and saline were injected in an identical fashion in the VEGF $_{165}$, LacZ, and saline groups, respectively.

Immunoprecipitation of receptor phosphorylation. To investigate the effect of VEGF-C overexpression on phosphorylation of VEGFR-2 and VEGFR-3, immunoprecipitation and Western blot analysis was performed in the mouse tail model. Lysis of tissues, immunoprecipitation, and Western blot analysis were performed as described $(31,33)$. Aliquots of protein extracts $(1 \mathrm{mg})$ were incubated for 2 hours at $4^{\circ} \mathrm{C}$ with $3 \mu \mathrm{g}$ of mAb against phosphotyrosine (Upstate Biotechnology Inc., Lake Placid, New York, USA), followed by incubation with $40 \mu \mathrm{l}$ of protein G-agarose beads (Roche Diagnostics $\mathrm{GmbH}$, Mannheim, Germany) overnight at $4^{\circ} \mathrm{C}$. Immunoprecipitates of tyrosine-phosphorylated proteins were separated by $7.5 \%$ SDS-PAGE and electrotransferred onto PVDF membranes. The membranes were immunoblotted overnight at $4^{\circ} \mathrm{C}$ with a rabbit polyclonal Ab against VEGFR-3 (1:500; Santa Cruz Biotechnology Inc., Santa Cruz, California, USA) or VEGFR-2 (1:500; Santa Cruz Biotechnology Inc.).
Immunohistochemistry and morphometric analysis. The skin from the bridge area was harvested 3 weeks after plasmid/saline injections. Skin sections were stained using a rat mAb against mouse VEGFR-3 (34) and a rabbit polyclonal $\mathrm{Ab}$ against the lymphatic marker lymphatic endothelial hyaluronan receptor-1 (LYVE-1), a receptor for hyaluronan and a homologue to the CD44 glycoprotein (35).

In double fluorescent immunohistochemistry of LYVE-1 and Ki-67, LYVE-1 staining was performed with the use of Texas red-streptavidin (NEN Life Science Products Inc., Boston, Massachusetts, USA), and Ki-67 staining was performed with rabbit polyclonal $\mathrm{Ab}$ against Ki-67 (Novocastra Laboratories Ltd., Newcastle, United Kingdom) and Cy2-conjugated goat anti-rabbit $\operatorname{IgG}$ (Jackson ImmunoResearch Laboratories Inc., West Grove, Pennsylvania, USA). Endothelial cells were identified by immunohistochemical staining for platelet endothelial cell adhesion molecule-1 (PECAM-1 or CD31) with a rat mAb against mouse CD31 (BD Biosciences, San Diego, California, USA) (36) in mouse tissues and with a mouse $\mathrm{mAb}$ against human $\mathrm{CD} 31$ in rabbit tissues.

Statistical analysis. All results were expressed as mean \pm SEM. Statistical analysis was performed with an unpaired Student $t$ test for comparisons between two groups and ANOVA followed by Scheffe's procedure for more than two groups. $P$ values $<0.05$ were considered to denote statistical significance.

\footnotetext{
Figure 4

(a) Gene transfer of phVEGF-C decreases lymphedema in a mouse tail model of lymphedema. Tail thickness was significantly greater in the operated tail than in the unoperated tail during the entire 5 weeks. In the VEGF-C group, compared with the saline, LacZ, and VEGF 165 groups, the tail thickness was significantly smaller at 3-5 weeks $\left({ }^{*} P<0.05\right)$. No-op, no operation. $(\mathbf{b}-\mathbf{m})$ phVEGF-C induces lymphangiogenesis in a mouse tail model of lymphedema. (b-k) Immunohistochemistry using markers of lymphatic endothelium, LVE-1 (b-f), and VEGFR-3 (g-k), in normal (b and $\mathbf{g}$ ) and operated ( 3 weeks after lymphedema surgery) skin sections from the saline ( $\mathbf{c}$ and $\mathbf{h}), \operatorname{LacZ}(\mathbf{d}$ and $\mathbf{i}), \operatorname{VEGF-C~(e~}$ and $\mathbf{j}$ ), and $\operatorname{VEGF}_{165}$ (f and $\mathbf{k}$ ) groups. Lymphatic vessels are seen as brown color (black arrows). Note the abundance of hyperplastic lymphatic vessels in phVEGF-C-transfected sections (e and $\mathbf{j}$ ). I and $\mathbf{m}$ show quantification of LYVE-1- and VEGFR-3-positive lymphatic vessels. Compared with normal and control (saline, LacZ, and VEGF 165 ) groups, the VEGF-C group showed significantly higher lymphatic vessel density. ${ }^{*} P<0.05$ vs. normal; ${ }^{*} P<0.01$ vs. LE-saline and LE-LacZ. Scale bar, $100 \mu \mathrm{m}$.
}
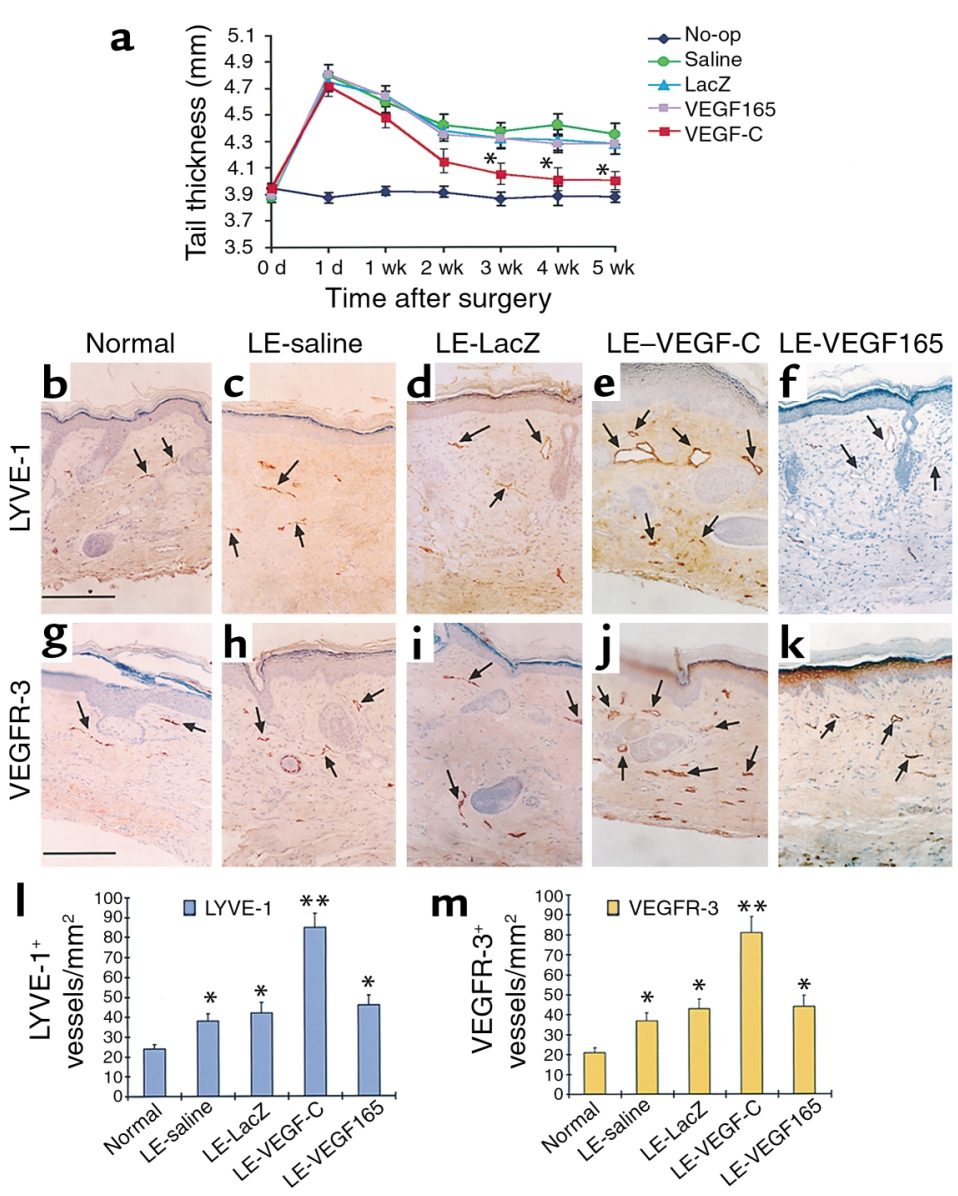

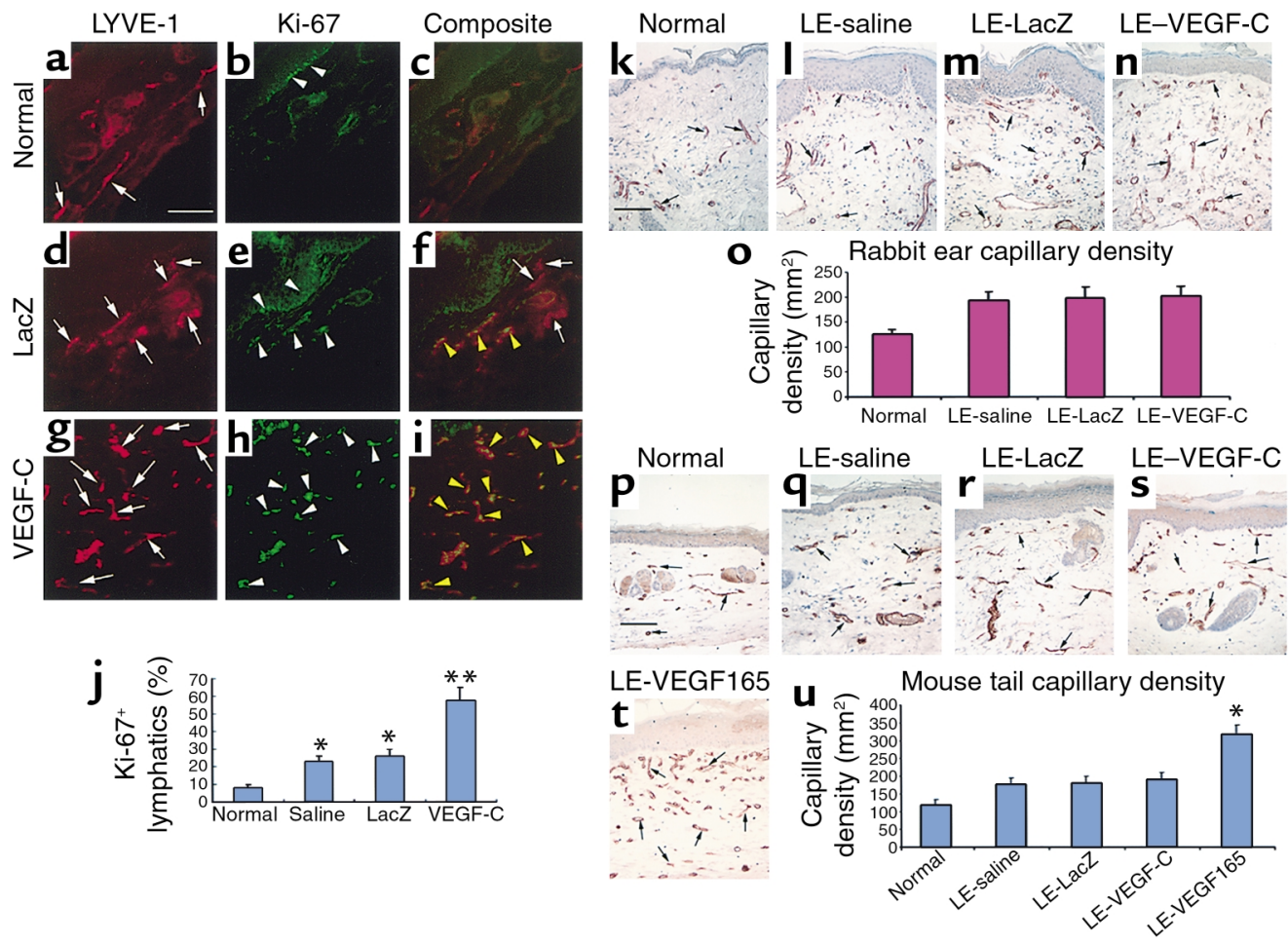

\section{Figure 5}

(a-j) phVEGF-C induces proliferation of lymphatic endothelial cells. Double immunohistochemistry using LYVE-1 and Ki-67 in active lymphangiogenesis site from skin sections. In $\mathbf{a}, \mathbf{d}$, and $\mathbf{g}$, LYVE-1 staining of lymphatic vessels (arrows) in the dermis. In $\mathbf{b}, \mathbf{e}$, and $\mathbf{h}$, green fluorescence (white arrowheads) depicts the nuclear staining of Ki-67. In c, f, and $\mathbf{i}$, double fluorescence (yellow arrowheads) demonstrates $\mathrm{Ki}-67^{+}$nuclei (green) in lymphatic vessels (red). Lymphatic vessels in normal skin (c) are shown negative for Ki-67. In the LacZ group, some of the lymphatic vessels contain Ki- $67^{+}$nuclei (f). White arrows in $\mathbf{f}$ show Ki-67- lymphatic vessels. In phVEGF-C-transfected skin, most of the LYE-1-positive lymphatic vessels are positive for Ki-67 (i), indicating that active cell division occurs in the lymphatic vessels. (j) Number of Ki- $67^{+}$nuclei are 2.5 times higher in the VEGF-C group. ${ }^{*} P<0.01$ compared with normal; ${ }^{*} P<0.01$ compared with saline and LacZ. Scale bar, $100 \mu \mathrm{m}$. (k-u) phVEGF-C does not increase capillary density in two animal models of lymphedema. Immunohistochemistry with CD31 (PECAM-1) in a rabbit ear $(\mathbf{k}-\mathbf{n})$ and a mouse tail ( $\mathbf{p}-\mathbf{t})$ model of lymphedema on skin sections from the normal (k and $\mathbf{p})$, saline (I and $\mathbf{q})$, LacZ ( $\mathbf{m}$ and $\mathbf{r}$ ), VEGF-C ( $\mathbf{n}$ and $\mathbf{s}$ ), and $\operatorname{VEGF}_{165}(\mathbf{t})$ groups. Vascular endothelial cells are stained red (black arrows). $\mathbf{o}$ and $\mathbf{u}$ show quantification of capillary density. Only the $\mathrm{VEGF}_{165}$ group in the mouse tail model demonstrated significantly higher capillary density than the other groups. ${ }^{*} P<0.01$ vs. saline, LacZ, and VEGF-C. Scale bar, $100 \mu \mathrm{m}$.

\section{Results}

VEGF-C gene therapy induces remission of lymphedema. Seeking to establish an appropriate animal model, initial experiments using young (6-8 months old) rabbits showed expedited regression of lymphedema such that we could not properly evaluate the effect of gene transfer. In the older rabbits (3-4 years old), a substantial degree of lymphedema developed and was sustained for more than 12 weeks.

To investigate the effect of phVEGF-C gene transfer on this lymphedema model, we measured ear thickness and volume over a 12 -week period. Ear thickness was consistently smaller in the VEGF-C group than in the saline group. The difference between the groups was statistically significant beginning at 2 weeks and was maintained for the duration of the study (2 weeks: $4.5 \pm 0.3$ vs. $5.4 \pm 0.2 \mathrm{~mm}, P<0.05 ; 3$ weeks: $3.9 \pm 0.2$ vs. $4.6 \pm 0.3 \mathrm{~mm}, P<0.05 ; 8$ weeks: $2.8 \pm 0.2$ vs. $3.6 \pm 0.3$ $\mathrm{mm}, P<0.05$; 10 weeks: $2.6 \pm 0.2$ vs. $3.5 \pm 0.3 \mathrm{~mm}$, $P<0.05$; 12 weeks: $2.4 \pm 0.2$ vs. $3.3 \pm 0.2 \mathrm{~mm}, P<0.01$ )
(Figure 1c). Similarly, ear volume was consistently smaller in the VEGF-C group than in the saline group for the duration of the study ( 2 weeks: $33.1 \pm 2.2$ vs. $38.2 \pm 1.5 \mathrm{ml}, P<0.05 ; 3$ weeks: $29.4 \pm 1.5$ vs. $34.1 \pm 1.0$ $\mathrm{ml}, P<0.05$; 4 weeks: $26.5 \pm 1.8$ vs. $31.7 \pm 1.7 \mathrm{ml}$, $P<0.05$; 8 weeks: $18.3 \pm 2.3$ vs. $26.3 \pm 1.9 \mathrm{ml}, P<0.05$; 10 weeks: $17.1 \pm 2.1$ vs. $25.5 \pm 1.8 \mathrm{ml}, P<0.01 ; 12$ weeks: $14.6 \pm 2.3$ vs. $24.5 \pm 1.3 \mathrm{ml}, P<0.01$ ) (Figure $1 \mathrm{~d}$ ). The VEGF-C group had significantly thinner skin than the saline group at 8 weeks $(2.8 \pm 0.2$ vs. $3.8 \pm 0.2$ $\mathrm{mm}, P<0.05)$.

Lymphoscintigraphy demonstrates enhanced lymphatic drainage after VEGF-C gene transfer. In normal ears, lymphatic flow assumes a linear pattern, and the draining LNs are clearly visible at the base of the skull. (Figure 2b). Imaging performed at day 1 after surgery showed successful surgical blockade of lymphatic egress in all animals (Figure 2, c and e). Follow-up lymphoscintigraphy at 4, 8, and 12 weeks showed dynamic changes of radiotracer clearance from the operated ears that was more efficient 
in the phVEGF-C-transfected ear than in the salineinjected ear. Images at 12 weeks revealed that the salineinjected ear still showed a dermal backflow pattern with faint visualization of LNs, while the phVEGF-C-transfected ear shows a linear pattern of lymphatic drainage and clear visualization of LNs (Figure 2, $\mathrm{d}$ and $\mathrm{f}$ ).

Quantification of lymphatic drainage (Figure 2, g and h) over the study period revealed consistently lower retention of radioactivity in the VEGF-C group than in the saline group; this achieved statistical significance at 8 weeks (radioactivity index, $3.8 \pm 0.4$ vs. $5.0 \pm 0.5$, $P<0.05$ ) and 12 weeks (radioactivity index, $2.2 \pm 0.3$ vs. $4.2 \pm 0.4, P<0.05$ ) (Figure 2i).

Transgene expression of phVEGF-C in a rabbit ear model. To assess transgene expression of injected phVEGF-C in ear skin, we performed Western blotting of VEGF-C protein. In our experiments, two different bands were detected using two anti-VEGF-C Ab's. A 58-kDa band corresponds to the earliest processed form, while a $31-\mathrm{kDa}$ band represents the major secreted form of VEGF-C polypeptides. Densitometric analysis of multiple experiments revealed that expression of the 58-kDa VEGF-C isoform in the phVEGF-C-transfected bridge was 2.6 times and 3.2 times higher than that in the saline-injected and the normal skin, respectively $(P<0.01)$ (Figure $3 a)$. Expression of the 31-kDa VEGF-C isoform in the phVEGF-C-transfected bridge was 3.6 times and 3.9 times higher than that in the saline-injected and the normal skin, respectively $(P<0.01)$ (Figure $3 \mathrm{~b})$.

Gene transfer of phVEGF-C increases VEGFR-3 expression. A partial 470-bp rabbit VEGFR-3 cDNA was cloned by RT-PCR using degenerate oligonucleotide primers (GenBank accession number AF453570). The amino acid sequence displayed $92.9 \%, 93.6 \%$, and $94.3 \%$ identity with human, bovine, and mouse VEGFR-3 (Figure $3 c$ ). We investigated VEGFR-3 expression using RT-PCR, revealing a nearly 1.7-fold induction of VEGFR-3 mRNA levels by VEGF-C compared with saline control $(P<0.01$, Figure $3 \mathrm{~g})$.

Gene transfer of phVEGF-C increases phosphorylation of $V E G F R-3$ in a mouse tail model. We investigated the effect of phVEGF-C gene transfer on the tyrosyl phosphorylation of VEGFR-3 and VEGFR-2 by immunoprecipitation with anti-phosphotyrosine $\mathrm{Ab}$ followed by Western blot analysis with anti-VEGFR-3 and anti-VEGFR-2 Ab's, respectively. Phosphorylated VEGFR-3 (195 kDa) in the phVEGF-C-transfected samples was 1.6 times and 1.8 times higher than in the samples from the saline and LacZ groups, respectively $(P<0.05)$ (Figure 3h). Gene transfer of phVEGF 165 did not increase phosphorylated VEGFR-3 compared with the controls. Phosphorylated VEGFR-2 in the phVEGF ${ }_{165}$-transfected sample was 2.0, 1.8, and 1.6 times higher (235-kDa band) than in the samples of the saline, LacZ, and VEGF-C groups, respectively $(P<0.05)$ (Figure $3 i)$. Phosphorylated VEGFR-2 was slightly higher in the phVEGF-Ctransfected samples than in the control groups (saline and LacZ) but was not statistically significant.
Gene transfer of phVEGF-C improves lymphedema in a mouse tail model. To determine whether the effect of phVEGF-C could be reproduced in another lymphedema model, similar experiments were performed in a mouse tail model (30) (Figure 4a). In the VEGF-C group, compared with the saline, LacZ, and VEGF $_{165}$ groups, the tail thickness was consistently smaller beginning at 3 weeks ( 3 weeks: $4.05 \pm 0.08$ vs. $4.37 \pm 0.07,4.32 \pm 0.08$, and $4.30 \pm 0.07 \mathrm{~mm}, P<0.05$; 4 weeks: $4.01 \pm 0.09 \mathrm{~mm}$ vs. $4.42 \pm 0.08,4.31 \pm 0.08$, and $4.28 \pm 0.07 \mathrm{~mm}, P<0.05$; 5 weeks: $4.00 \pm 0.07$ $\mathrm{mm}$ vs. $4.35 \pm 0.08,4.28 \pm 0.08$, and $4.28 \pm 0.08 \mathrm{~mm}$, $P<0.05)$, respectively.

Gene transfer of phVEGF-C promotes lymphatic vessel growth in a mouse tail model. The VEGF-C group showed higher density of LYVE-1-positive lymphatic vessels than the other groups (VEGF-C, $85 \pm 7$ per $\mathrm{mm}^{2}$; saline, $38 \pm 4$ per $\mathrm{mm}^{2}$; LacZ, $42 \pm 5$ per $\mathrm{mm}^{2} ; \mathrm{VEGF}_{165}, 46 \pm 5$ per $\mathrm{mm}^{2}, P<0.01$ ) (Figure 4, b-f and l). Skin sections stained with VEGFR-3 Ab showed similar results (VEGF-C, $81 \pm 8$ per $\mathrm{mm}^{2}$; saline, $37 \pm 4$ per $\mathrm{mm}^{2}$; LacZ, $43 \pm 5$ per $\mathrm{mm}^{2} ; \mathrm{VEGF}_{165}, 44 \pm 5$ per $\mathrm{mm}^{2}, P<0.01$ ) (Figure $4, \mathrm{~g}-\mathrm{k}$ and $\mathrm{m}$ ). After phVEGF-C transfection, lymphatic vessels appeared hyperplastic (Figure 4, e and j). In sections at 3-week follow-up, the number of lymphatic vessels containing $\mathrm{Ki}-67^{+}$nuclei was 2.5 times higher in the VEGF-C group than in the saline or LacZ groups (VEGF-C, $58 \pm 7$ per $\mathrm{mm}^{2}$; saline, $23 \pm 3$ per $\mathrm{mm}^{2}$; LacZ, $26 \pm 4$ per $\mathrm{mm}^{2}, P<0.01$ ) (Figure 5, a-j).

Blood capillary density analysis. Rabbit ear and mouse tail skins were stained for an endothelial cell marker, CD31 (36). In the rabbit lymphedema model, capillary density was not significantly different among the saline $\left(193 \pm 18\right.$ per $\left.\mathrm{mm}^{2}\right), \operatorname{LacZ}\left(198 \pm 22\right.$ per $\left.\mathrm{mm}^{2}\right)$, or VEGF-C $\left(201 \pm 20\right.$ per $\mathrm{mm}^{2}$ ) groups (Figure 5, k-o). Similar findings were observed in the capillary density of operated mouse tail groups (saline, $172 \pm 18$ per $\mathrm{mm}^{2}$; LacZ, $181 \pm 19$ per $\mathrm{mm}^{2}$; VEGF-C, $189 \pm 20$ per $\mathrm{mm}^{2}, P$ value not significant) (Figure $\left.5 \mathrm{~b}, \mathrm{~F}-\mathrm{K}\right)$. However, the $\mathrm{VEGF}_{165}$ group $\left(302 \pm 27\right.$ per $\left.\mathrm{mm}^{2}\right)$ showed significantly higher capillary density than the saline, LacZ, or VEGF-C groups $(P<0.01)$.

\section{Discussion}

Chronic lymphedema is a disabling condition characterized by thickening of the skin due to fibrofatty deposition in underlying tissues as well as disfiguring swelling of affected limbs. In most cases of secondary lymphedema in humans, depletion of lymphatic vessels is the culprit in its pathogenesis (1-4). Here we show that phVEGF-C gene therapy, by promoting lymphangiogenesis, favorably modulates all the phenotypic changes associated with secondary lymphedema. We believe the present study is the first to document improvement in the clinical and pathologic features of lymphedema resulting from enhancement of lymphatic drainage by phVEGF-C gene therapy.

In two animal models, we demonstrate significant attenuation of lymphedema by phVEGF-C gene transfer. 
The effect prevailed over the chronic phase as well as the acute phase of lymphedema. This improvement was also confirmed in histologic sections, which reflect chronic fibrofatty changes more accurately. Prevention or reduction of fibrotic change is one of the most important goals of therapy for lymphedema, since this secondary change can drive lymphedema into a vicious cycle by increasing interstitial solid pressure (by fibrofatty deposition) and thus collapsing already reduced or impaired lymphatic vessels $(3-5,24,25)$. That the improvement in the physical indices of lymphedema was the actual result of improved lymphatic drainage was documented by quantitative lymphoscintigraphy. Although VEGF-C plasmid transgene expression is usually limited to less than 30 days (27), our results indicate that once the lymphatic connection is reestablished, the recovery of drainage function can be maintained.

VEGF-C protein expression was documented in situ after phVEGF-C gene transfer. We detected the partially processed form $(58 \mathrm{kDa})$ and major secreted form $(31 \mathrm{kDa})$ of VEGF-C $(37,38)$ after local gene delivery. VEGF-C is produced as a $61-\mathrm{kDa}$ prepropeptide form which undergoes multistep proteolytic maturation. The secreted $31-\mathrm{kDa}$ form predominantly activates VEGFR-3 (37). VEGFR-3 mRNA expression was very low in normal skin, slightly higher in the saline-injected experimental ears, and strongly upregulated following phVEGF-C gene transfer. We also directly measured the number of lymphatic vessels using lymphatic-specific markers. LYVE-1 or VEGFR-3 staining confirmed augmentation of lymphangiogenesis in phVEGF-C-transfected mouse tails. The hyperplastic nature of proliferating lymphatic vessels was consistent with previous reports $(19,22)$. Ki-67 staining documented that proliferating lymphatic endothelial cells exist in more than half the lymphatic vessels in phVEGF-C-transfected skin, suggesting a potent lymphangiogenic effect of phVEGF-C.

As VEGF-C is also known to activate VEGFR-2 and thus to induce angiogenesis in vitro and in ischemic tissues $(15,32)$, we evaluated capillary density from both animal models and found it was slightly higher in the VEGF-C group but not significantly different from that of the saline or LacZ control groups. The apparent absence of fully processed 21-kDa product of VEGF-C, which has potent angiogenic activity, and thus the weak activation of VEGFR-2 phosphorylation, could explain the lack of obvious angiogenesis in these animal models. These findings are compatible with previous reports that claimed no discernible angiogenesis in transgenic mice overexpressing VEGF-C in the skin (19) and in normal mouse skin that was transfected with adeno-VEGF-C (22). Physiologic function of any ligand is dependent on the temporal and spatial expression of its specific receptors. In the case of VEGF-A-induced angiogenesis, the absence of ischemia-induced regional upregulation of VEGFR-2 has been shown to result in nullification of the angiogenic effect of transient overexpression of VEGF-A (39,
40). To further address the concern that increased angiogenesis might improve lymphedema, we investigated the effect of VEGF-A ( $\left.\mathrm{phVEGF}_{165}\right)$ plasmid gene transfer in the mouse tail model and found that augmenting angiogenesis but not lymphangiogenesis did not improve lymphedema.

The potential clinical relevance and limitations of our study derive from certain features of the design and the findings. First, the models are pathophysiologically similar to the secondary forms of human lymphedema. The models we used do not represent the entire spectrum of lymphedema found in humans, especially the primary form of lymphedema, which is an inherited developmental disorder of the lymphatic system. However, pathophysiologically our models are approximations of secondary lymphedema, which comprises most cases of lymphedema and results primarily from surgical removal of lymphatic vessels and lymph nodes in industrialized countries (1-4). Additionally, in our models, we used gene therapy in an acute/subacute stage of lymphedema. Therefore, whether this gene therapy can be effective in chronic cases is uncertain. As there are concerns about the potential enhancement of tumor growth and metastasis by VEGF-C in tumor models (41), we need to consider the potential advantages and dangers of using local VEGF-C therapy in patients with lymphedema caused by cancer treatment. This issue can be resolved after performing experiments adopting tumor implantation and treatment such as a combination of surgery, chemotherapy or radiation, and local VEGF-C gene transfer. Third, the approach of gene therapy using naked plasmid DNA (phVEGF-C) has been used in early clinical trials and has an accumulating record of safety (42). Finally, to the best of our knowledge, this study represents the first experimental proof of a beneficial effect of VEGF-C gene therapy on lymphedema per se. Our findings clearly indicate a favorable effect of phVEGF-C-induced lymphangiogenesis on lymphedema and thus represent a novel therapeutic paradigm for the treatment of this otherwise difficult-to-manage condition.

\section{Acknowledgments}

This paper is dedicated to Jeffrey M. Isner, who passed away on October 31,2001. We would like to gratefully acknowledge his inspirational leadership. We gratefully acknowledge M. Neely, I. Johnson, and T. Shiojima for their excellent secretarial assistance. This study was supported in part by NIH grants HL-53354, HL60911, HL-63414, HL-63695, and HL-66957, and by the Shaughnessy Center for Clinical Genetics, Boston, Massachusetts, USA. Young-sup Yoon is the recipient of a fellowship from the American Heart Association, New England Affiliate.

\footnotetext{
1. Browse, N.L. 1986. The diagnosis and management of primary lymphedema. J. Vasc. Surg. 3:181-184.

2. de Almeida, A.B., and Freedman, D.O. 1999. Epidemiology and immunopathology of bancroftian filariasis. Microbes Infect. 1:1015-1022.

3. Szuba, A., and Rockson, S.G. 1998. Lymphedema: classification, diagnosis and therapy. Vasc. Med. 3:145-156.
} 
4. Ko, D.S., Lerner, R., Klose, G., and Cosimi, A.B. 1998. Effective treatment of lymphedema of the extremities. Arch. Surg. 133:452-458.

5. Slavin, S.A., Upton, J., Kaplan, W.D., and Van den Abbeele, A.D. 1997. An investigation of lymphatic function following free-tissue transfer. Plast. Reconstr. Surg. 99:730-741; discussion 742-743.

6. Drinker, C.K., Field, M.J., and Homans J. 1934. The experimental production of edema and elephantiasis as a result of lymphatic obstruction. Am. J. Physiol. 108:509-516.

7. Clodius, L. 1976. Experimental lymphedema and therapeutic concepts. Acta Chir. Plast. 18:113-116.

8. Casley-Smith, J.R., Clodius, L., and Foldi, M. 1977. Experimental blood vascular and lymphatic occlusion in the rabbit ear and the effect of benzopyrones. Arzneimittelforschung. 27:379-382.

9. Lee-Donaldson, L., et al. 1999. Refinement of a rodent model of peripheral lymphedema. Lymphology. 32:111-117.

10. Leak, L.V., and Jones, M. 1994. Lymphangiogenesis in vitro: formation of lymphatic capillary-like channels from confluent monolayers of lymphatic endothelial cells. In Vitro Cell. Dev. Biol. Anim. 30A:512-518.

11. Oh, S.J., et al. 1997. VEGF and VEGF-C: specific induction of angiogenesis and lymphangiogenesis in the differentiated avian chorioallantoic membrane. Dev. Biol. 188:96-109.

12. Aprelikova, O., et al. 1992. FLT4, a novel class III receptor tyrosine kinase in chromosome 5q33-qter. Cancer Res. 52:746-748.

13. Galland, F., et al. 1992. Chromosomal localization of FLT4, a novel receptor-type tyrosine kinase gene. Genomics. 13:475-478.

14. Lee, J., et al. 1996. Vascular endothelial growth factor-related protein: a ligand and specific activator of the tyrosine kinase receptor Flt4. Proc. Natl. Acad. Sci. U. S. A. 93:1988-1992.

15. Joukov, V., et al. 1996. A novel vascular endothelial growth factor, VEGF-C, is a ligand for the Flt4 (VEGFR-3) and KDR (VEGFR-2) receptor tyrosine kinases. EMBO J. 15:290-298.

16. Kaipainen, A., et al. 1995. Expression of the fms-like tyrosine kinase 4 gene becomes restricted to lymphatic endothelium during development. Proc. Natl. Acad. Sci. U. S. A. 92:3566-3570.

17. Kukk, E., et al. 1996. VEGF-C receptor binding and pattern of expression with VEGFR-3 suggests a role in lymphatic vascular development. Development. 122:3829-3837.

18. Baumgartner, I., et al. 1998. Constitutive expression of phVEGF165 after intramuscular gene transfer promotes collateral vessel development in patients with critical limb ischemia. Circulation. 97:1114-1123.

19. Jeltsch, M., et al. 1997. Hyperplasia of lymphatic vessels in VEGF-C transgenic mice. Science. 276:1423-1425.

20. Ferrell, R.E., et al. 1998. Hereditary lymphedema: evidence for linkage and genetic heterogeneity. Hum. Mol. Genet. 7:2073-2078.

21. Karkkainen, M.J., et al. 2000. Missense mutations interfere with VEGFR-3 signalling in primary lymphoedema. Nat. Genet. 25:153-159.

22. Enholm, B., et al. 2001. Adenoviral expression of vascular endothelial growth factor-C induces lymphangiogenesis in the skin. Circ. Res. 88:623-629.

23. Karkkainen, M.J., et al. 2001. A model for gene therapy of human hereditary lymphedema. Proc. Natl. Acad. Sci. U. S. A. 98:12677-12682.
24. Piller, N.B., and Clodius, L. 1978. Lymphoedema of the rabbit ear following partial and complete lymphatic blockade; its effects on fibrotic development, enzyme types and their activity levels. Br. J. Exp. Pathol. 59:319-326.

25. Fu, K., Izquierdo, R., Vandevender, D., Warpeha, R.L., and Fareed, J. 1998. Transplantation of lymph node fragments in a rabbit ear lymphedema model: a new method for restoring the lymphatic pathway. Plast. Reconstr. Surg. 101:134-141.

26. Tsurumi, Y., et al. 1997. Reciprocal relation between VEGF and NO in the regulation of endothelial integrity. Nat. Med. 3:879-886.

27. Schratzberger, P., et al. 2001. Reversal of experimental diabetic neuropathy by VEGF gene transfer. J. Clin. Invest. 107:1083-1092.

28. Finnerty, H., et al. 1993. Molecular cloning of murine FLT and FLT4. Oncogene. 8:2293-2298.

29. Galland, F., et al. 1993. The FLT4 gene encodes a transmembrane tyrosine kinase related to the vascular endothelial growth factor receptor. Oncogene. 8:1233-1240.

30. Slavin, S.A., Van den Abbeele, A.D., Losken, A., Swartz, M.A., and Jain, R.K. 1999. Return of lymphatic function after flap transfer for acute lymphedema. Ann. Surg. 229:421-427.

31. Schratzberger, P., et al. 2000. Favorable effect of VEGF gene transfer on ischemic peripheral neuropathy. Nat. Med. 6:405-413.

32. Witzenbichler, B., et al. 1998. Vascular endothelial growth factor-C (VEGF-C/VEGF-2) promotes angiogenesis in the setting of tissue ischemia. Am. J. Pathol. 153:381-394.

33. Brogi, E., et al. 1996. Hypoxia-induced paracrine regulation of vascular endothelial growth factor receptor expression. J. Clin. Invest. 97:469-476.

34. Kubo, H., et al. 2000. Involvement of vascular endothelial growth factor receptor-3 in maintenance of integrity of endothelial cell lining during tumor angiogenesis. Blood. 96:546-553.

35. Banerji, S., et al. 1999. LYVE-1, a new homologue of the CD44 glycoprotein, is a lymph-specific receptor for hyaluronan. J. Cell Biol. 144:789-801.

36. Rivard, A., et al. 1999. Age-dependent impairment of angiogenesis. Circulation. 99:111-120.

37. Joukov, V., et al. 1997. Proteolytic processing regulates receptor specificity and activity of VEGF-C. EMBO J. 16:3898-3911.

38. Pepper, M.S., Mandriota, S.J., Jeltsch, M., Kumar, V., and Alitalo, K. 1998. Vascular endothelial growth factor (VEGF)-C synergizes with basic fibroblast growth factor and VEGF in the induction of angiogenesis in vitro and alters endothelial cell extracellular proteolytic activity. J. Cell. Pbysiol. 177:439-452.

39. Takeshita, S., et al. 1994. Therapeutic angiogenesis. A single intraarterial bolus of vascular endothelial growth factor augments revascularization in a rabbit ischemic hind limb model. J. Clin. Invest. 93:662-670.

40. Isner, J.M., et al. 1996. Arterial gene transfer for therapeutic angiogenesis in patients with peripheral artery disease. Hum. Gene Ther. 7:959-988.

41. Skobe, M., et al. 2001. Induction of tumor lymphangiogenesis by VEGF-C promotes breast cancer metastasis. Nat. Med. 7:192-198.

42. Isner, J.M., Vale, P.R., Symes, J.F., and Losordo, D.W. 2001. Assessment of risks associated with cardiovascular gene therapy in human subjects. Circ. Res. 89:389-400. 